\title{
Research Article \\ On the Well Posedness and Refined Estimates for the Global Attractor of the TYC Model
}

\author{
Rana D. Parshad ${ }^{1}$ and Juan B. Gutierrez ${ }^{2}$ \\ ${ }^{1}$ Department of Mathematics and Computer Science, Clarkson University, Potsdam, NY 13676, USA \\ ${ }^{2}$ Mathematical Biosciences Institute, Ohio State University, Columbus, OH 43210, USA
}

Correspondence should be addressed to Rana D. Parshad, rparshad@clarkson.edu

Received 14 July 2010; Accepted 2 November 2010

Academic Editor: Sandro Salsa

Copyright (c) 2010 R. D. Parshad and J. B. Gutierrez. This is an open access article distributed under the Creative Commons Attribution License, which permits unrestricted use, distribution, and reproduction in any medium, provided the original work is properly cited.

The Trojan Y Chromosome strategy (TYC) is a theoretical method for eradication of invasive species. It requires constant introduction of artificial individuals into a target population, causing a shift in the sex ratio that ultimately leads to local extinction. In this work we demonstrate the existence of a unique weak solution to the infinite dimensional TYC system. Furthermore, we obtain improved estimates on the upper bounds for the Hausdorff and fractal dimensions of the global attractor of the TYC system, via the use of weighted Sobolev spaces. These results confirm that the TYC eradication strategy is a sound theoretical method of eradication of invasive species in a spatial setting. It also provides a solid ground for experiments in silico and validates the use of the TYC strategy in vivo.

\section{Introduction}

An exotic species is a species that resides outside its native habitat. When it causes some sort of measurable damage, it is often referred to as invasive. The recent globalization process has expedited the pace at which exotic species are introduced into new environments. Once established, these species can be extremely difficult to manage and almost impossible to eradicate $[1,2]$. Studies have indicated that the losses caused by invasive species could be as much as $\$ 120$ billion/year by 2004 [3]. The effect of these invaders is thus devastating [4]. Current approaches for controlling exotic fish species are limited to general chemical control methods applied to small water bodies and/or small isolated populations that kill native fish in addition to the target fish [5]. For example, the piscicide Rotenone has been used to eradicate exotic fish, but at the expense of killing all the endogenous fish, making it necessary to restock native fish from other sources $[1,2]$.

A genetic strategy to cause extinction of invasive species was proposed by Gutierrez and Teem [6]. This strategy is relevant to species amenable to sex reversal and with an XY sexdetermination system, in which males are the heterogametic sex (carrying one $\mathrm{X}$ chromosome 
and one $\mathrm{Y}$ chromosome, $\mathrm{XY}$ ) and females are the homogametic sex (carrying two $\mathrm{X}$ chromosomes, $\mathrm{XX}$ ). The strategy relies on the fact that variations in the sex chromosome number can be produced through genetic manipulation, for example, a normal and fertile male bearing two Y chromosomes (supermale, YY) [7-10]. Also hormone treatments can be used to reverse the sex, resulting in a feminized $Y Y$ supermale $[5,11,12]$.

The eradication strategy requires adding a sex-reversed "Trojan" female individual bearing two $\mathrm{Y}$ chromosomes, that is, feminized supermales $(r)$, at a constant rate $\mu$ to a target population of an invasive species, containing normal females and males denoted as $f$ and $m$, respectively. Matings involving the introduced $r$ generate a disproportionate number of males over time. The higher incidence of males decrease the female to male ratio. Ultimately, the number of $f$ decline to zero, causing local extinction. This theoretical method of eradication is known as Trojan Y Chromosome (TYC) strategy.

The original model considered by Gutierrez and Teem was an ODE model. Spatial spread is ubiquitous in aquatic settings and was thus considered by Gutierrez et al. [13], resulting in a PDE model. In [14], we considered the PDE model and showed the existence of a global attractor for the system, which is $H^{2}(\Omega)$ regular, attracting orbits uniformly in the $L^{2}(\Omega)$ metric. We showed that this attractor supports a state, in which the female population is driven to zero, thus resulting in local extinction. Recall the TYC model with spatial spread takes the following form [14]:

$$
\begin{gathered}
\frac{\partial f}{\partial t}=D \Delta f+\frac{1}{2} f m \beta L-\delta f,\left.\quad f\right|_{\partial \Omega}=0 \\
\frac{\partial m}{\partial t}=D \Delta m+\left(\frac{1}{2} f m+\frac{1}{2} r m+f s\right) \beta L-\delta m,\left.\quad m\right|_{\partial \Omega}=0, \\
\frac{\partial s}{\partial t}=D \Delta s+\left(\frac{1}{2} r m+r s\right) \beta L-\delta s,\left.\quad s\right|_{\partial \Omega}=0, \\
\frac{\partial r}{\partial t}=D \Delta r+\mu-\delta r,\left.\quad r\right|_{\partial \Omega}=0 .
\end{gathered}
$$

Here, $\Omega \subset R^{3}$ is a bounded domain. Also

$$
L=1-\left(\frac{f+m+r+s}{K}\right)
$$

where $K$ is the carrying capacity of the ecosystem, $D$ is a diffusivity coefficient, $\delta$ is a birth coefficient (i.e., what proportion of encounters between males and females result in progeny), and $\delta$ is a death coefficient (i.e., what proportion of the population is dying at any given moment). We assume initial data is positive and in $L^{2}(\Omega)$. At the outset we would like to point out that the difficulty in analyzing (1.1)-(1.4) lies in the nonlinear terms $L f m, L((1 / 2) f m+$ $(1 / 2) r m+f s)$ and $L((1 / 2) r m+r s)$. See [15] for a PDE dealing with similar nonlinearities, albeit in the setting of a fluid-saturated porous medium. We will also assume positivity of solutions as negative $f, m, r, s$ do not make sense in the biological context. We also provide a rigoros proof to this end. 
In the current paper we will show that the TYC model, (1.1)-(1.4), possesses a unique weak solution $(f, m, r, s)$. By this we mean that there exist $(f, m, r, s)$ such that the following is satisfied in the distributional sense:

$$
\begin{gathered}
\frac{d}{d t}\langle f, v\rangle+D\langle\nabla f, \nabla v\rangle+\delta\langle f, v\rangle=\left\langle\frac{1}{2} f m \beta L, v\right\rangle \\
\frac{d}{d t}\langle m, v\rangle+D\langle\nabla m, \nabla v\rangle+\delta\langle m, v\rangle=\left\langle\left[\frac{1}{2} f m+\frac{1}{2} r m+f s\right] \beta L, v\right\rangle \\
\frac{d}{d t}\langle r, v\rangle+D\langle\nabla s, \nabla v\rangle+\delta\langle s, v\rangle=\left\langle\left[\frac{1}{2} r m+r s\right] \beta L, v\right\rangle \\
\frac{d}{d t}\langle s, v\rangle+D\langle\nabla s, \nabla v\rangle+\delta\langle s, v\rangle=\langle\mu, v\rangle .
\end{gathered}
$$

Here, $\langle\cdot\rangle$ is the standard inner product in $L^{2}(\Omega)$. Furthermore the above hold for all $v \in$ $H_{0}^{1}(\Omega)$. Our main result is summarized in the following theorem.

Theorem 1.1. Consider the Trojan Y Chromosome model, (1.1)-(1.4). There exists a unique weak solution $(f, m, r, s)$ to the system for positive initial data in $L^{2}(\Omega)$, such that

$$
\begin{gathered}
(f, m, r, s) \in C\left([0, T] ; L^{2}(\Omega)\right) \cap L^{\infty}\left(0, T ; L^{2}(\Omega)\right) \cap L^{2}\left(0, T ; H_{0}^{1}(\Omega)\right), \\
\left(\frac{\partial f}{\partial t}, \frac{\partial m}{\partial t}, \frac{\partial r}{\partial t}, \frac{\partial s}{\partial t}\right) \in L^{2}\left(0, T ; H^{-1}(\Omega)\right)
\end{gathered}
$$

for all $T>0$. Furthermore, $(f, m, r, s)$ are continuous with respect to initial data.

Our strategy to prove the above is as follows: we first derive a priori estimates for the $f, m, r, s$ variables. We then show existence of a solution to (1.1). Note, showing existence of a solution to (1.1) requires a priori estimates on $(m, r, s)$ also. The key here is Lemma 4.1 which enables convergence of the nonlinear term $L f m$. Next we show uniqueness of the solution to (1.1). The procedure to show existence and uniqueness of solutions to (1.2)(1.4) follow similarly. We then consider the question of sharpening the upper bounds on the Hausdorff and fractal dimension of the global attractor for the system, derived in [14]. This constitutes our second main result, Theorem 7.2. Lastly, we offer some concluding remarks. In all estimates made hence, forth, $C$ is a generic constant that can change in its value from line to line and sometimes within the same line if so required.

\section{A Bound in $L^{\infty}(\Omega)$}

The biology of the system dictates that the solutions are bounded in the supremum norm by the carrying capacity. We now provide a proof via a maximum principle argument. 
Lemma 2.1. Consider the Trojan Y Chromosome model, (1.1)-(1.4). The solutions $f, m, r, s$ of the system are bounded as follows:

$$
\begin{aligned}
& |f|_{\infty} \leq K, \\
& |m|_{\infty} \leq K, \\
& |s|_{\infty} \leq K, \\
& |r|_{\infty} \leq K .
\end{aligned}
$$

Proof. The proof relies heavily on the form of the nonlinearity in the system. We concentrate on the nonlinear term in (1.1),

$$
F(f, m, r, s)=f m\left(1-\frac{f+m+r+s}{K}\right) .
$$

The analysis for the other terms is similar. As is biologically viable, we assumes $f, m, r$, and $s$ are always positive, thus, we have

$$
f>0, \quad m>0, \quad r>0, \quad s>0 .
$$

Assuming positive initial data, $f_{0}>0, m_{0}>0, r_{0}>0$, and $s_{0}>0$, the solution at later times remains positive. In order to prove this let us assume the contrary, that is $f_{0}>0, m_{0}>0, r_{0}>0$, and $s_{0}>0$, but say $f$ can become negative at a later time. Consider an interior minimum point in the parabolic cylider $\Omega \times[0, T]$, that is some $\left(x^{*}, t^{*}\right)$, such that $f$ attains a minimum there, and that $f\left(x^{*}, t^{*}\right)<0, m\left(x^{*}, t^{*}\right)<0, r\left(x^{*}, t^{*}\right)<0$, and $s\left(x^{*}, t^{*}\right)<0$. Under this setting, from standard calculus, we have

$$
\frac{\partial f}{\partial t}\left(x^{*}, t^{*}\right)=0, \quad \Delta f\left(x^{*}, t^{*}\right) \geq 0
$$

furthermore,

$$
\begin{gathered}
-\delta f\left(x^{*}, t^{*}\right)>0, \\
\beta f\left(x^{*}, t^{*}\right) m\left(x^{*}, t^{*}\right)\left(1-\frac{f\left(x^{*}, t^{*}\right)+m\left(x^{*}, t^{*}\right)+r\left(x^{*}, t^{*}\right)+s\left(x^{*}, t^{*}\right)}{K}\right)>0 .
\end{gathered}
$$


Thus from (1.1), we have

$$
\begin{aligned}
\frac{\partial f}{\partial t}\left(x^{*}, t^{*}\right)= & 0 \\
= & \Delta f\left(x^{*}, t^{*}\right)-\delta f\left(x^{*}, t^{*}\right) \\
& +\beta f\left(x^{*}, t^{*}\right) m\left(x^{*}, t^{*}\right)\left(1-\frac{f\left(x^{*}, t^{*}\right)+m\left(x^{*}, t^{*}\right)+r\left(x^{*}, t^{*}\right)+s\left(x^{*}, t^{*}\right)}{K}\right) \\
> & 0+0+0 \\
= & 0 .
\end{aligned}
$$

This is clearly a contradiction. Thus even at an interior minimum $f>0$, hence $f>0$ everywhere else. The same argument can be applied on the equations describing the $m, r$, and $s$ variables. Actually the equation for $r$ is exactly solvable and is seen to be positive. Thus our assumption via (2.3) is feasible. Thus we proceed with our proof via maximum principle. Despite not biologically viable, assume for purposes of analysis that

$$
f \geq K \geq 1, \quad m \geq K \geq 1 .
$$

We now define the positive and negative parts of $(f-K)$ as

$$
\begin{aligned}
& (f-K)_{+}(x)= \begin{cases}f-K, & f>K \\
0, & \text { otherwise }\end{cases} \\
& (f-K)_{-}(x)= \begin{cases}f-K, & f<K \\
0, & \text { otherwise. }\end{cases}
\end{aligned}
$$

We now multiply $(1.1)$ by $(f-K)_{+}(x)$ and integrate by parts to yield

$$
\frac{d}{d t}\left|(f-K)_{+}\right|_{2}^{2}+\left|\nabla(f-K)_{+}\right|_{2}^{2}+\delta\left|(f-K)_{+}\right|_{2}^{2} \leq \int_{\Omega} F(f, m, r, s)(f-K)_{+}(x) d \mathbf{x} .
$$

When $f<K$ the right-hand side is zero. When $f>K$, assuming $f \geq K+\epsilon$ where $\epsilon>0$, and $m>k$ via (2.3), we have

$$
\begin{aligned}
\int_{\Omega} F(f, m, r, s)(f-K)_{+}(x) d \mathbf{x} & =\int_{\Omega} f m\left(1-\frac{f+m+r+s}{K}\right)(f-K)_{+}(x) d \mathbf{x} \\
& \leq \int_{\Omega}(K+\epsilon) K\left(1-\frac{f+m+r+s}{K}\right)(\epsilon) d \mathbf{x} \\
& \leq \int_{\Omega}(K+\epsilon) K\left(1-\frac{2 K+2 \delta}{K}\right)(\epsilon) d \mathbf{x} \\
& \leq|\Omega|(K+\epsilon)^{2} \epsilon\left(-1-\frac{2 \delta}{K}\right) \\
& \leq|\Omega|(K+\epsilon)^{2} \epsilon\left(-\frac{2 \delta}{K}\right) \\
& \leq 0 .
\end{aligned}
$$


Hence, via Poincaré's Inequality, we obtain

$$
\frac{d}{d t}\left|(f-K)_{+}\right|_{2}^{2}+(C+\delta)\left|(f-K)_{+}\right|_{2}^{2} \leq 0
$$

Application of Gronwall's Lemma now yields

$$
\left|(f-K)_{+}\right|_{2}^{2} \leq e^{-(C+\delta) t}\left|\left(f_{0}-K\right)_{+}\right|_{2}^{2} .
$$

We can now consider $t \rightarrow \infty$ to yield

$$
(f-K)_{+}=0
$$

The same argument on the negative part of $f$ yields,

$$
(f-K)_{-}=0 .
$$

Since the positive and negative parts of $f$ can be no more than $K$, we obtain

$$
|f|_{\infty} \leq K
$$

The same technique works on $m, s$, and $r$ and is trivially seen to be bounded from the form of (1.4).

\section{A Priori Estimates}

\subsection{A Priori Estimates for $f_{n}$}

In order to prove the well posedness we follow the standard approach of projecting onto a finite dimensional subspace. This reduces the PDE to a finite dimensional system of ODE's. It is on this truncated system that we make a priori estimates. Essentially The truncation for $f$ takes the form

$$
f_{n}(t)=\sum_{j=1}^{n} f_{n j}(t) w_{j}
$$

Here $w_{j}$ are the eigenfunctions of the negative Laplacian, so $-\Delta w_{i}=\lambda_{i} w_{i}$. A similar truncation can be performed for $m, r$ and $s$. Thus, essentially the following holds for all $1 \leq j \leq n$,

$$
\begin{gathered}
\frac{\partial f_{n}}{\partial t}=D \Delta f_{n}+P_{n}\left(F\left(f_{n}, m_{n}, r_{n}, s_{n}\right)\right)-\delta f_{n} \\
f_{n}(0)=P_{n}\left(f_{0}\right) .
\end{gathered}
$$


Here $P_{n}$ is the projection onto the space of the first $n$ eigenvectors. Note in general

$$
\left\langle f_{n}, P_{n}\left(F\left(f_{n}\right)\right)\right\rangle=\left\langle P_{n}\left(f_{n}\right), F\left(f_{n}\right)\right\rangle=\left\langle f_{n}, F\left(f_{n}\right)\right\rangle .
$$

We multiply (3.2) by $f_{n}$ and integrate by parts over $\Omega$. We thus obtain

$$
\frac{1}{2} \frac{d\left|f_{n}\right|_{2}^{2}}{d t}=-D\left|\nabla f_{n}\right|_{2}^{2}+\frac{\beta}{2}\left[\int_{\Omega} m_{n} f_{n}^{2} d \mathbf{x}-\int_{\Omega} m_{n} f_{n}^{2} \frac{f_{n}+m_{n}+r_{n}+s_{n}}{K} d \mathbf{x}\right]-\delta\left|f_{n}\right|_{2}^{2} .
$$

Via the positivity of $f_{n}, m_{n}, r_{n}, s_{n}$, and $K$ it follows that

$$
\int_{\Omega} m_{n} f_{n}^{2} \frac{f_{n}+m_{n}+r_{n}+s_{n}}{K} d \mathbf{x} \geq \int_{\Omega} m_{n} f_{n}^{2} \frac{f_{n}}{K} d \mathbf{x}
$$

This estimate is used in (3.5) to yield

$$
\frac{1}{2} \frac{d\left|f_{n}\right|_{2}^{2}}{d t}+D\left|\nabla f_{n}\right|_{2}^{2}+\delta\left|f_{n}\right|_{2}^{2}+\frac{\beta}{2 K} \int_{\Omega} m_{n} f_{n}^{3} d \mathbf{x} \leq \frac{\beta}{2} \int_{\Omega} m_{n} f_{n}^{2} d \mathbf{x}
$$

We now use Young's Inequality to obtain

$$
\frac{1}{2} \frac{d\left|f_{n}\right|_{2}^{2}}{d t}+D\left|\nabla f_{n}\right|_{2}^{2}+\delta\left|f_{n}\right|_{2}^{2}+\frac{\beta}{2 K} \int_{\Omega} m_{n} f_{n}^{3} d \mathbf{x} \leq \frac{\beta}{2 K} \int_{\Omega} m_{n} f_{n}^{3} d \mathbf{x}+\frac{\beta K^{2}}{2} \int_{\Omega} m_{n} d \mathbf{x}
$$

Using

$$
\left|m_{n}\right|_{\infty} \leq|m|_{\infty} \leq K,
$$

we obtain the following

$$
\frac{1}{2} \frac{d\left|f_{n}\right|_{2}^{2}}{d t}+D\left|\nabla f_{n}\right|_{2}^{2}+\delta\left|f_{n}\right|_{2}^{2} \leq \frac{\beta K^{3}}{2}|\Omega|
$$

The use of Poincaré's Inequality yields

$$
\frac{d\left|f_{n}\right|_{2}^{2}}{d t}+(C D+\delta)\left|f_{n}\right|_{2}^{2} \leq \beta K^{3}|\Omega|
$$

Now, we can apply Gronwall's Lemma to yield

$$
\left|f_{n}(t)\right|_{2}^{2} \leq e^{-(C D+\delta) t}\left|f_{0}\right|_{2}^{2}+\frac{\beta K^{3}|\Omega|}{C D+\delta} \leq C, \quad \forall t \geq 0 .
$$


On the other hand we can integrate (3.10) from 0 to $T$ to obtain

$$
\frac{1}{2}\left|f_{n}(T)\right|_{2}^{2}+D \int_{0}^{T}\left|\nabla f_{n}\right|_{2}^{2} d t+\delta \int_{0}^{T}\left|f_{n}\right|_{2}^{2} d t \leq \int_{0}^{T} \beta K^{3}|\Omega| d t+\left|f_{n}(0)\right|_{2}^{2} .
$$

This immediately yields

$$
\begin{aligned}
\int_{0}^{T}\left|\nabla f_{n}\right|_{2}^{2} d t & \leq \int_{0}^{T} \beta K^{3}|\Omega| d t+\left|f_{n}(0)\right|_{2}^{2} \\
& \leq \int_{0}^{T} \beta K^{3}|\Omega| d t+|f(0)|_{2}^{2} \\
& \leq C .
\end{aligned}
$$

Thus, via (3.12) and (3.14), we obtain

$$
\begin{aligned}
& f_{n} \in L^{\infty}\left(0, T ; L^{2}(\Omega)\right), \\
& f_{n} \in L^{2}\left(0, T ; H_{0}^{1}(\Omega)\right) .
\end{aligned}
$$

\subsection{Estimate for the Time Derivative of $f_{n}$}

We multiply (3.2) by a $w \in H_{0}^{1}(\Omega)$ to yield

$$
\left(\frac{\partial f_{n}}{\partial t}, w\right)=-D\left\langle\nabla f_{n}, \nabla w\right\rangle+\left\langle F\left(f_{n}, m_{n}, r_{n}, s_{n}\right), P_{n}(w)\right\rangle-\delta\left\langle f_{n}, w\right\rangle .
$$

We estimate the nonlinear term as follows:

$$
\begin{aligned}
\left(F\left(f_{n}\right), P_{n}(w)\right) & =\int_{\Omega} m_{n} f_{n}\left(1-\frac{f_{n}+m_{n}+r_{n}+s_{n}}{K}\right) P_{n}(w) d \mathbf{x} \\
& \leq \int_{\Omega} m_{n} f_{n} P_{n}(w) d \mathbf{x} \\
& \leq\left|m_{n}\right|_{\infty} \int_{\Omega} f_{n} P_{n}(w) d \mathbf{x} \\
& \leq K\left|f_{n}\right|_{4}\left|P_{n}(w)\right|_{4 / 3} \\
& \leq C\left|f_{n}\right|_{4}|w|_{H_{0}^{1}} .
\end{aligned}
$$

This follows via the compact embedding of $H_{0}^{1}(\Omega) \hookrightarrow L^{4 / 3}(\Omega)$. Thus, we have

$$
\left|\frac{\partial f_{n}}{\partial t}\right|_{H^{-1}(\Omega)}^{2} \leq\left|f_{n}\right|_{4}^{2} .
$$


Integrating both sides of the above in the time interval $[0, T]$ yields

$$
\int_{0}^{T}\left|\frac{\partial f_{n}}{\partial t}\right|_{H^{-1}(\Omega)}^{2} d t \leq \int_{0}^{T}\left|f_{n}\right|_{4}^{2} d t \leq C \int_{0}^{T}\left|\nabla f_{n}\right|_{2}^{2} d t \leq C .
$$

This follows from the derived estimate via (3.16) and the compact embedding of $H_{0}^{1}(\Omega) \hookrightarrow$ $L^{4}(\Omega)$. Thus, we obtain

$$
\frac{\partial f_{n}}{\partial t} \in L^{2}\left(0, T ; H^{-1}(\Omega)\right)
$$

We can now via (3.15) and (3.16) extract a subsequence $f_{n_{j}}$ such that

$$
\begin{aligned}
& f_{n_{j}} \stackrel{*}{\rightarrow} f \quad \text { in } L^{\infty}\left(0, T ; L^{2}(\Omega)\right), \\
& f_{n_{j}} \rightarrow f \quad \text { in } L^{2}\left(0, T ; H_{0}^{1}(\Omega)\right), \\
& f_{n_{j}} \longrightarrow f \quad \text { in } L^{2}\left(0, T ; L^{2}(\Omega)\right) .
\end{aligned}
$$

The convergence in the last equation follows via the compact embedding of $H_{0}^{1}(\Omega) \hookrightarrow L^{2}(\Omega)$.

\subsection{A Priori Estimates for $m, r$, and $s$}

The a priori estimates for $m, r$ and $s$ are very similar to the estimates for $f$. We omit the details here and present the results.

The truncation for $m$ satisfies the following a priori estimates:

$$
\begin{gathered}
m_{n} \in L^{\infty}\left(0, T ; L^{2}(\Omega)\right), \\
m_{n} \in L^{2}\left(0, T ; H_{0}^{1}(\Omega)\right), \\
\frac{\partial s_{n}}{\partial t} \in L^{2}\left(0, T ; H^{-1}(\Omega)\right) .
\end{gathered}
$$

We can now extract a subsequence $m_{n_{j}}$ such that

$$
\begin{aligned}
& m_{n_{j}} \stackrel{*}{\rightarrow} s \text { in } L^{\infty}\left(0, T ; L^{2}(\Omega)\right), \\
& m_{n_{j}} \rightarrow s \text { in } L^{2}\left(0, T ; H_{0}^{1}(\Omega)\right), \\
& m_{n_{j}} \longrightarrow s \text { in } L^{2}\left(0, T ; L^{2}(\Omega)\right) .
\end{aligned}
$$


The last inequality follows via the compact embedding of

$$
H_{0}^{1}(\Omega) \hookrightarrow L^{2}(\Omega)
$$

The truncation for $s$ satisfies the following a priori estimates:

$$
\begin{gathered}
s_{n} \in L^{\infty}\left(0, T ; L^{2}(\Omega)\right), \\
s_{n} \in L^{2}\left(0, T ; H_{0}^{1}(\Omega)\right), \\
\frac{\partial s_{n}}{\partial t} \in L^{2}\left(0, T ; H^{-1}(\Omega)\right) .
\end{gathered}
$$

We can now extract a subsequence $s_{n_{j}}$ such that

$$
\begin{aligned}
& s_{n_{j}} \stackrel{*}{*} s \quad \text { in } L^{\infty}\left(0, T ; L^{2}(\Omega)\right), \\
& s_{n_{j}} \rightarrow s \quad \text { in } L^{2}\left(0, T ; H_{0}^{1}(\Omega)\right), \\
& s_{n_{j}} \longrightarrow s \text { in } L^{2}\left(0, T ; L^{2}(\Omega)\right) .
\end{aligned}
$$

The last inequality follows via the compact embedding of

$$
H_{0}^{1}(\Omega) \hookrightarrow L^{2}(\Omega)
$$

The truncation for $r$ satisfies the following a priori estimates:

$$
\begin{gathered}
r_{n} \in L^{\infty}\left(0, T ; L^{2}(\Omega)\right), \\
r_{n} \in L^{2}\left(0, T ; H_{0}^{1}(\Omega)\right), \\
\frac{\partial r_{n}}{\partial t} \in L^{2}\left(0, T ; H^{-1}(\Omega)\right) .
\end{gathered}
$$

We can now extract a subsequence $r_{n_{j}}$ such that

$$
\begin{aligned}
& r_{n_{j}} \stackrel{*}{\rightarrow} \quad \text { in } L^{\infty}\left(0, T ; L^{2}(\Omega)\right), \\
& r_{n_{j}} \rightarrow r \quad \text { in } L^{2}\left(0, T ; H_{0}^{1}(\Omega)\right), \\
& r_{n_{j}} \longrightarrow r \quad \text { in } L^{2}\left(0, T ; L^{2}(\Omega)\right) .
\end{aligned}
$$


The last inequality follows via the compact embedding of

$$
H_{0}^{1}(\Omega) \hookrightarrow L^{2}(\Omega)
$$

\section{Existence of Solution}

\subsection{Preliminaries}

We recast (1.1) in the following form:

$$
\frac{\partial f}{\partial t}=D \Delta f+F(f, r, m, s)-\delta f,\left.\quad f\right|_{\partial \Omega}=0
$$

Here,

$$
F(f, m, r, s)=\frac{\beta}{2}\left(1-\frac{f+m+r+s}{K}\right) f m
$$

Note that the key element in proving the existence will be to show convergence of the nonlinear term $F\left(f_{n_{j}}, m_{n_{j}}, r_{n_{j}}, s_{n_{j}}\right)$ to $F(f, m, r, s)$. To this end we state the following lemma,

Lemma 4.1. Consider the non linear terms $F\left(f_{1}, m_{1}, r_{1}, s_{1}\right)$ and $F\left(f_{2}, m_{2}, r_{2}, s_{2}\right)$ as defined via (4.2). The following estimate for their difference holds

$$
\left|F\left(f_{1}, m_{1}, r_{1}, s_{1}\right)-F\left(f_{2}, m_{2}, r_{2}, s_{2}\right)\right|_{2} \leq C\left(\left|f_{1}-f_{2}\right|_{2}+\left|m_{1}-m_{2}\right|_{2}+\left|s_{1}-s_{2}\right|_{2}+\left|r_{1}-r_{2}\right|_{2}\right) .
$$

Proof. Via (4.2), we have that

$$
\begin{aligned}
\left(F\left(f_{1}, m_{2}, r_{2}, s_{2}\right)-F\left(f_{2}, m_{2}, r_{2}, s_{2}\right)\right) \\
=f_{1} m_{1}-f_{2} m_{2}-\left(f_{1}^{2} m_{1}-f_{2}^{2} m_{2}\right)-\left(m_{1}^{2} f_{1}-m_{2}^{2} f_{2}\right) \\
\quad+f_{1} m_{1} r_{1}-f_{2} m_{2} r_{2}+f_{1} m_{1} s_{1}-f_{2} m_{2} s_{2} \\
=f_{1}\left(m_{1}-m_{2}\right)+m_{2}\left(f_{1}-f_{2}\right)-\left(f_{1}^{2}\left(m_{1}-m_{2}\right)+m_{2}\left(f_{1}^{2}-f_{2}^{2}\right)\right) \\
\quad-\left(m_{1}^{2}\left(f_{1}-f_{2}\right)+f_{2}\left(m_{1}^{2}-m_{2}^{2}\right)\right)+m_{1} r_{1}\left(f_{1}-f_{2}\right)+f_{2} m_{2}\left(r_{1}-r_{2}\right) \\
\quad+f_{2} r_{1}\left(m_{1}-m_{2}\right)+m_{1} s_{1}\left(f_{1}-f_{2}\right)+f_{2} m_{2}\left(s_{1}-s_{2}\right) \\
\quad+f_{2} s_{1}\left(m_{1}-m_{2}\right) .
\end{aligned}
$$

(We supress the dependence of the right-hand side on the constant $\beta / 2$ for convenience.) 
This follows from standard algebraic manipulation. Application of Holder's and Minkowski's inequalities yield

$$
\begin{aligned}
\mid F\left(f_{1},\right. & \left.m_{2}, r_{2}, s_{2}\right)-\left.F\left(f_{2}, m_{2}, r_{2}, s_{2}\right)\right|_{2} \\
\leq & \left|f_{1}\right|_{\infty}\left|m_{1}-m_{2}\right|_{2}+\left|m_{2}\right|_{\infty}\left|f_{1}-f_{2}\right|_{2}+\left|f_{1}\right|_{\infty}^{2}\left|m_{1}-m_{2}\right|_{2} \\
& +\left|m_{2}\right|_{\infty}\left|f_{1}+f_{2}\right|_{\infty}\left|f_{1}-f_{2}\right|_{2}+\left|m_{1}\right|_{\infty}^{2}\left|f_{1}-f_{2}\right|_{2} \\
& +\left|f_{2}\right|_{\infty}\left|m_{1}+m_{2}\right|_{\infty}\left|m_{1}-m_{2}\right|_{2} \\
& +\left|m_{1}\right|_{\infty}\left|r_{1}\right|_{\infty}\left|f_{1}-f_{2}\right|_{2}+\left|f_{2}\right|_{\infty}\left|m_{2}\right|_{\infty}\left|r_{1}-r_{2}\right|_{2}+\left|r_{1}\right|_{\infty}\left|f_{2}\right|_{\infty}\left|m_{1}-m_{2}\right|_{2} \\
& +\left|s_{1}\right|_{\infty}\left|m_{1}\right|_{\infty}\left|f_{1}-f_{2}\right|_{2}+\left|f_{2}\right|_{\infty}\left|m_{2}\right|_{\infty}\left|s_{1}-s_{2}\right|_{2}+\left|s_{1}\right|_{\infty}\left|f_{2}\right|_{\infty}\left|m_{1}-m_{2}\right|_{2} \\
\leq & C\left(\left|f_{1}-f_{2}\right|_{2}+\left|m_{1}-m_{2}\right|_{2}+\left|s_{1}-s_{2}\right|_{2}+\left|r_{1}-r_{2}\right|_{2}\right) .
\end{aligned}
$$

\subsection{Passage to Weak Limit}

As, we have made the a priori estimates on the truncations, we will attempt to pass to the weak limit, as is the standard practice. We will focus on (1.1). Recall via Galerkin truncation we are seeking an approximate solution of the form

$$
f_{n}(t)=\sum_{j=1}^{n} f_{n j}(t) w_{j}
$$

such that, for each $1 \leq j \leq n$, and for all $\phi \in C_{0}^{\infty}(0, T)$, the following holds:

$$
\begin{gathered}
\left\langle\frac{d f_{n}}{d t}, \phi w_{j}\right\rangle+D\left\langle\nabla f_{n_{j}}, \nabla w_{j} \phi(t)\right\rangle+\delta\left\langle f_{n_{j}}, \phi(t) w_{j}\right\rangle=\left\langle F\left(f_{n_{j}}\right), \phi w_{j}\right\rangle, \\
f_{n}(0)=P_{n}\left(f_{0}\right) .
\end{gathered}
$$

(Here and henceforth we assume $F\left(f_{n_{j}}\right)=P_{n}\left(F\left(f_{n_{j}}\right)\right)$, where $P_{n}$ is the projection operator onto the first $n$ eigenvectors). Upon passage to the weak limit of (4.7), we will have obtained

$$
\left\langle\frac{d f}{d t}, w_{j}\right\rangle+D\left\langle\nabla f, \nabla w_{j}\right\rangle+\delta\left\langle f, w_{j}\right\rangle=\left\langle F(f), w_{j}\right\rangle
$$

This will imply the existence of a weak solution $f$ to (1.1). We proceed as follows. Consider a $\phi \in C_{0}^{\infty}(0, T)$. We multiply (4.7) by $\phi(t)$ and integrate by parts in time to yield

$$
\begin{aligned}
-\int_{0}^{T}\left\langle f_{n_{j}}, \phi^{\prime}(t) w_{j}\right\rangle d t= & -D \int_{0}^{T}\left\langle\nabla f_{n_{j}}, \nabla w_{j} \phi(t)\right\rangle d t+\int_{0}^{T}\left\langle F\left(f_{n_{j}}\right), \phi(t) w_{j}\right\rangle d t \\
& -\delta \int_{0}^{T}\left\langle f_{n_{j}}, \phi(t) w_{j}\right\rangle d t .
\end{aligned}
$$

We will first show convergence of the nonlinear term. This is stated via the following lemma. 
Lemma 4.2. Consider the nonlinear term $F(f, m, r, s)$ as defined via (4.2). The following convergence result holds:

$$
\lim _{j \rightarrow \infty} \int_{0}^{T} \int_{\Omega} F\left(f_{n_{j}}, m_{n_{j}}, s_{n_{j}}, r_{n_{j}}\right) \phi(t) w_{j} d \mathbf{x} d t=\int_{0}^{T} \int_{\Omega} F(f, m, s, r) \phi(t) w_{j} d \mathbf{x} d t
$$

for all $\phi \in C_{0}^{\infty}(0, T)$.

Proof. Consider

$$
\begin{aligned}
\lim _{j \rightarrow \infty} \int_{0}^{T} \int_{\Omega} F\left(f_{n_{j}}\right) \phi(t) w_{j} d \mathbf{x} d t-\int_{0}^{T} \int_{\Omega} F(f) \phi(t) w_{j} d \mathbf{x} d t \mid \\
\leq C \int_{0}^{T} \int_{\Omega}\left|\left(F\left(f_{n_{j}}\right), \phi w_{j}\right)-\left(F(f), \phi w_{j}\right)\right|^{2} d \mathbf{x} d t \\
\leq C|\phi|_{\infty}\left|w_{j}\right|_{\infty} \int_{0}^{T} \int_{\Omega}\left|F(f)-F\left(f_{n_{j}}\right)\right|^{2} d \mathbf{x} d t \\
\leq C \int_{0}^{T}\left(\left|f-f_{n_{j}}\right|_{2}^{2}+\left|m-m_{n_{j}}\right|_{2}^{2}+\left|r-r_{n_{j}}\right|_{2}^{2}+\left|s-s_{n_{j}}\right|_{2}^{2}\right) d t \\
\leq C\left(\left|f-f_{n_{j}}\right|_{L^{2}\left(0, T ; L^{2}\right)}+\left|m-m_{n_{j}}\right|_{L^{2}\left(0, T ; L^{2}\right)}+\left|s-s_{n_{j}}\right|_{L^{2}\left(0, T ; L^{2}\right)}\right) \\
\quad+C\left(\left|r-r_{n_{j}}\right|_{L^{2}\left(0, T ; L^{2}\right)}\right) \\
\leq C(0+0+0+0) \\
=0 .
\end{aligned}
$$

This follows via Lemma 4.1 and because, we have demonstrated

$$
\begin{aligned}
& f_{n_{j}} \longrightarrow f \quad \text { in } L^{2}\left(0, T ; L^{2}(\Omega)\right), \\
& m_{n_{j}} \longrightarrow m \quad \text { in } L^{2}\left(0, T ; L^{2}(\Omega)\right), \\
& s_{n_{j}} \longrightarrow s \quad \text { in } L^{2}\left(0, T ; L^{2}(\Omega)\right), \\
& r_{n_{j}} \longrightarrow r \quad \text { in } L^{2}\left(0, T ; L^{2}(\Omega)\right) .
\end{aligned}
$$


Thus the convergence of the nonlinear term has been established. Now, taking the limit as $j \rightarrow \infty$ in (4.10), we obtain

$$
\begin{aligned}
\lim _{j \rightarrow \infty} \int_{0}^{T}\left\langle f_{n_{j}}, \phi^{\prime}(t) w_{j}\right\rangle d t+D \int_{0}^{T}\left\langle\nabla f_{n_{j}}, \nabla w_{j} \phi(t)\right\rangle d t \\
\quad+\delta \int_{0}^{T}\left\langle f_{n_{j}}, \phi w_{j}\right\rangle d t-\int_{0}^{T}\left\langle F\left(f_{n_{j}}\right), \phi w_{j}\right\rangle d t \\
=\int_{0}^{T}\left\langle f, \phi^{\prime}(t) w_{j}\right\rangle d t+D \int_{0}^{T}\left\langle\nabla f, \nabla w_{j} \phi\right\rangle d t \\
\quad+\delta \int_{0}^{T}\left\langle f, \phi w_{j}\right\rangle d t-\int_{0}^{T}\left\langle F(f), \phi w_{j}\right\rangle d t \\
=0 .
\end{aligned}
$$

The last term on the right-hand side can be bounded as follows

$$
\begin{aligned}
\int_{0}^{T}\left\langle F(f), \phi w_{j}\right\rangle d t & \leq C|\phi|_{\infty} \int_{0}^{T} \int_{\Omega}\left|f^{2}\right|\left|w_{j}\right| d t \\
& \leq C|\phi|_{\infty}\left|w_{j}\right|_{2}|f|_{L^{2}\left(0, T ; L^{4}(\Omega)\right)} \\
& \leq C|\phi|_{\infty}\left|w_{j}\right|_{H_{0}^{1}(\Omega)}|f|_{L^{2}\left(0, T ; H_{0}^{1}(\Omega)\right)} \\
& \leq C\left|w_{j}\right|_{H_{0}^{1}(\Omega)} .
\end{aligned}
$$

This follows by the compact embedding of $H_{0}^{1}(\Omega) \hookrightarrow L^{4}(\Omega) \hookrightarrow L^{2}(\Omega)$. This implies that, we have continuity with respect to $w_{j}$. Thus, we obtain that for any $v \in H_{0}^{1}(\Omega)$ the following holds

$$
-\int_{0}^{T}\left\langle f, \phi^{\prime}(t) v\right\rangle d t+D \int_{0}^{T}\langle\nabla f, \nabla v \phi(t)\rangle d t+\delta \int_{0}^{T}\langle f, \phi(t) v\rangle d t=\int_{0}^{T}\langle F(f), \phi(t) v\rangle d t
$$

This yields the existence of an $f$ such that the following is true in a distributional sense

$$
\frac{d}{d t}\langle f, v\rangle+D\langle\nabla f, \nabla v\rangle+\delta\langle f, v\rangle=\langle F(f), v\rangle, \quad \forall v \in H_{0}^{1}(\Omega)
$$

In other words there exists a weak solution $f$ to (1.1). Since

$$
\begin{gathered}
f \in L^{\infty}\left(0, T ; L^{2}(\Omega)\right) \cap L^{2}\left(0, T ; H_{0}^{1}(\Omega)\right), \\
\frac{\partial f}{\partial t} \in L^{2}\left(0, T ; H^{-1}(\Omega)\right),
\end{gathered}
$$


it follows via standard PDE theory, see [16, 17], that

$$
f \in C\left([0, T] ; L^{2}(\Omega)\right) .
$$

This establishes that the solution belongs to the requisite functional spaces.

\subsection{Continuity with Respect to Initial Data and Uniqueness of Solutions}

We now show continuity with respect to initial data of the solution via the following lemma.

Lemma 4.3. Consider the Trojan Y Chromosome model. For positive initial data in $L^{2}(\Omega)$, any weak solution $(f, m, s, r)$ of the Trojan $Y$ Chromosome model is continuous with respect to initial data, that is,

$$
f(0)=f_{0}, \quad m(0)=m_{0}, \quad s(0)=s_{0}, \quad r(0)=r_{0} .
$$

Proof. We will show the details for $f$, and the other variables follow suit accordingly. We take a test function $\phi \in C^{1}[0, T]$ such that

$$
\phi(0)=1, \quad \phi(T)=0 .
$$

With this choice of $\phi(t)$ in (4.17), we integrate the first term twice by parts to yield

$$
\begin{gathered}
-\int_{0}^{T}\left\langle f, \phi^{\prime}(t) v\right\rangle d t+D \int_{0}^{T}\langle\nabla f, \nabla v \phi(t)\rangle d t+\delta \int_{0}^{T}\langle f, \phi(t) v\rangle d t \\
=\langle f(0), v\rangle+D \int_{0}^{T}\langle\nabla f, \nabla v \phi(t)\rangle d t+\delta \int_{0}^{T}\langle f, \phi(t) v\rangle d t .
\end{gathered}
$$

Note that the truncation satisfies

$$
\begin{array}{r}
\int_{0}^{T}\left\langle f_{n_{j}}, \phi^{\prime}(t) v\right\rangle d t+D \int_{0}^{T}\left\langle\nabla f_{n_{j}}, \nabla v \phi(t)\right\rangle d t+\delta \int_{0}^{T}\left\langle f_{n_{j}}, \phi(t) v\right\rangle d t \\
=\left\langle f_{n_{j}}(0), v\right\rangle+D \int_{0}^{T}\left\langle\nabla f_{n_{j}}, \nabla v \phi(t)\right\rangle d t+\delta \int_{0}^{T}\left\langle f_{n_{j}}, \phi(t) v\right\rangle d t
\end{array}
$$

Thus, taking the limit as $j \rightarrow \infty$ in (4.28) just as done earlier yields

$$
\begin{gathered}
-\int_{0}^{T}\left\langle f, \phi^{\prime}(t) v\right\rangle d t+D \int_{0}^{T}\langle\nabla f, \nabla v \phi(t)\rangle d t+\delta \int_{0}^{T}\langle f, \phi(t) v\rangle d t \\
=\left\langle f_{0}, v\right\rangle+D \int_{0}^{T}\langle\nabla f, \nabla v \phi(t)\rangle d t+\delta \int_{0}^{T}\langle f, \phi(t) v\rangle d t .
\end{gathered}
$$


Thus, we obtain

$$
\langle f(0), v\rangle=\left\langle f_{0}, v\right\rangle, \quad \forall v \in H_{0}^{1}(\Omega) .
$$

This yields

$$
f(0)=f_{0},
$$

as is required.

We now state the uniqueness result via the following lemma.

Lemma 4.4. Consider the Trojan $Y$ Chromosome model. For positive initial data in $L^{2}(\Omega)$ any weak solution $(f, m, s, r)$ of the Trojan $Y$ Chromosome model is unique.

Proof. We work out the case for the $f$ variable, uniqueness for the others follow similarly. We consider the difference of two solutions $f_{1}$ and $f_{2}$ to (1.1). We denote

$$
w=f_{1}-f_{2},
$$

and $w$ satisfies the following equation:

$$
\begin{gathered}
\frac{d w}{d t}-D \Delta w+\delta w=F\left(f_{1}\right)-F\left(f_{2}\right), \\
w(0)=f_{1}(0)-f_{2}(0)=0 .
\end{gathered}
$$

We can multiply (4.28) by $w$ and integrate by parts over $\Omega$ to yield

$$
\frac{d|w|_{2}^{2}}{d t}+D|\nabla w|_{2}^{2}+\delta|w|_{2}^{2}=\int_{\Omega}\left(F\left(f_{1}\right)-F\left(f_{2}\right)\right) w d \mathbf{x}
$$

Via the uniform $L^{2}$ estimates on $m, r, s$, see [14], and Lemma 4.1, we obtain

$$
\frac{d|w|_{2}^{2}}{d t}+D|\nabla w|_{2}^{2}+\delta|w|_{2}^{2} \leq C\left|f_{1}-f_{2}\right|_{2}|w|_{2} \leq C K|w|_{2}^{2} .
$$

This yields

$$
\frac{d|w|_{2}^{2}}{d t}+D|\nabla w|_{2}^{2}+\delta|w|_{2}^{2}-(C K)|w|_{2}^{2} \leq 0
$$

Now using Poincarés Inequality, we obtain,

$$
\frac{d|w|_{2}^{2}}{d t}+(D+\delta-C)|w|_{2}^{2} \leq 0
$$


The use of Gronwall's Lemma yields that for any $t>0$ the following estimate holds:

$$
|w(t)|_{2}^{2} \leq e^{-(D+\delta-C) t}|w(0)|_{2}^{2} \leq 0
$$

Equation (4.17) in conjunction with Lemma 4.4 yields Theorem 1.1.

\section{Weighted Sobolev Spaces}

The purpose of this section is to introduce weighted Sobolev spaces into the framework of our present problem. We will show that $r$ given by (1.4), remains bounded in the norms of these spaces. This will enable us to state a theorem about the existence of weak solution in the weighted spaces. This in turn will entail making refined estimates on the dimension of the global attractor for TYC system, when the phase space is a weighted Sobolev space. This will be achieved via the elegant technique of projecting the trace operator onto a weighted Sobolev space. We first make certain requisite definitions.

Definition 5.1. The weighted Sobolev space $W_{\omega(x)}^{k, p}$, with weight function $\omega(x)$, is defined to be the space consisting of all functions $u$ such that

$$
\left(\sum_{|\alpha| \leq k} \int_{\Omega}\left|D^{\alpha} u\right|^{p} \omega(x) d x\right)^{1 / p}<\infty
$$

Remark 5.2. Here, $D^{\alpha}$ is the $\alpha$ th weak derivative of $u$. In particular, we are interested in the following spaces for our application:

$$
\begin{gathered}
L_{\omega}^{2}(\Omega)=\left\{u:\left(\int_{\Omega} \omega(x)|u|^{2} d \mathbf{x}\right)^{1 / 2}<\infty\right\}, \\
H_{0, \omega}^{1}(\Omega)=\left\{u:|u|_{2, \omega}+|\nabla u|_{2, \omega}<\infty\right\} .
\end{gathered}
$$

Also, we denote $\left(\int_{\Omega} \omega(x)|u|^{2} d \mathbf{x}\right)^{1 / 2}=|u|_{2, \omega}$. We define $H_{\omega}^{-1}(\Omega)$ to be the dual of $H_{0, \omega}^{1}(\Omega)$.

\subsection{Estimates for $r$ in Weighted Sobolev Spaces}

Recall the equation for $r$

$$
\frac{\partial r}{\partial t}=D \Delta r-\delta r+\mu,\left.\quad r\right|_{\partial \Omega}=0
$$


We choose $\omega(x)=e^{\mu x}, \mu>0$, multiply (5.3) by $r e^{\mu x}$, and integrate by parts over $\Omega$ to yield

$$
\begin{aligned}
\frac{1}{2} \frac{d}{d t} \int_{\Omega}|r|^{2} e^{\mu x} d \mathbf{x}= & -D \int_{\Omega}|\nabla r|^{2} e^{\mu x} d \mathbf{x}-D \int_{\Omega} \nabla r \cdot \nabla\left(r e^{\mu x}\right) d \mathbf{x}-\delta \int_{\Omega}|r|^{2} e^{\mu x} d \mathbf{x} \\
& +\mu \int_{\Omega} r e^{\mu x} d \mathbf{x} \\
\leq & -D \int_{\Omega}|\nabla r|^{2} e^{\mu x} d \mathbf{x}+\frac{D}{2} \int_{\Omega}|\nabla r|^{2} e^{\mu x} d \mathbf{x}+\frac{\mu^{2}}{2} \int_{\Omega}|r|^{2} e^{\mu x} d \mathbf{x} \\
& -\delta \int_{\Omega}|r|^{2} e^{\mu x} d \mathbf{x}+\mu \int_{\Omega} r e^{\mu x} d \mathbf{x} \\
\leq & -\frac{D}{2} \int_{\Omega}|\nabla r|^{2} e^{\mu x} d x-\delta \int_{\Omega}|r|^{2} e^{\mu x} d \mathbf{x}+C\left(\frac{\mu^{2} K^{2}}{2}+\mu K\right)|\Omega|
\end{aligned}
$$

These follow via integration by parts, the estimate $|r|_{\infty} \leq K$, and the Cauchy-Schwartz inequality. Thus, we obtain

$$
\frac{1}{2} \frac{d}{d t}|r|_{2, \omega}^{2}+\frac{D}{2}|\nabla r|_{2, \omega}^{2}+\delta|r|_{2, \omega}^{2} \leq C\left(\frac{\mu^{2} K^{2}}{2}+\mu K\right)|\Omega|
$$

The use of Poincaire's Inequality gives us

$$
\frac{1}{2} \frac{d|r|_{2, \omega}^{2}}{d t}+\left(\frac{D}{2}+\delta\right)|\nabla r|_{2, \omega}^{2} \leq C\left(\frac{\mu^{2} K^{2}}{2}+\mu K\right)|\Omega|
$$

Now, we can apply the Gronwall Lemma to yield

$$
|r(t)|_{2, \omega}^{2} \leq e^{-(C D+\delta) t}\left|r_{0}\right|_{2, \omega}^{2}+\frac{\mu^{2} K^{2} / 2+\mu K}{C D+\delta}, \quad \forall t \geq 0
$$

On the other hand we can integrate (5.5) from 0 to $T$ to obtain

$$
\frac{1}{2}|r(T)|_{2, \omega}^{2}+\frac{D}{2} \int_{0}^{T}|\nabla r|_{2, \omega}^{2} d t+\delta \int_{0}^{T}|r|_{2, \omega}^{2} d t \leq \int_{0}^{T}\left(\frac{\mu^{2} K^{2}}{2}+\mu K\right)|\Omega| d t .
$$

This immediately yields

$$
\int_{0}^{T}|\nabla r|_{2, \omega}^{2} d t \leq \int_{0}^{T}\left(\frac{\mu^{2} K^{2}}{2}+\mu K\right)|\Omega| d t
$$


Thus, via (5.7) and (5.9), we have that

$$
\begin{aligned}
& |r|_{L^{\infty}\left(0, T ; L_{\omega}^{2}(\Omega)\right)} \leq C<\infty, \\
& |r|_{L^{2}\left(0, T ; H_{0, \omega}^{1}(\Omega)\right)} \leq C<\infty .
\end{aligned}
$$

\subsection{Estimate for the Time Derivative of $r$ in Weighted Sobolev Space}

We multiply (5.3) by a $w \in H_{0, \omega}^{1}(\Omega)$ to yield

$$
\begin{gathered}
\left\langle\frac{\partial r}{\partial t}, w\right\rangle_{2, \omega}=-D\langle\nabla r, \nabla w\rangle_{2, \omega}-\delta\langle r, w\rangle_{2, \omega}+\langle w, \mu\rangle_{2, \omega^{\prime}} \\
\left|\frac{\partial r}{\partial t}\right|_{H_{\omega}^{-1}(\Omega)} \leq \mu|w|_{2, \omega} .
\end{gathered}
$$

Integrating both sides in time from 0 to $T$ yields

$$
\int_{0}^{T}\left|\frac{\partial r}{\partial t}\right|_{H_{\omega}^{-1}(\Omega)}^{2} d t \leq \mu \int_{0}^{T}\left(|w|_{2, \omega}^{2}\right) d t
$$

Because of the estimate via (5.11) and the embedding of

$$
H_{0, \omega}^{1}(\Omega) \hookrightarrow L_{\omega}^{2}(\Omega),
$$

we have

$$
\frac{\partial r}{\partial t} \in L^{2}\left(0, T ; H_{\omega}^{-1}(\Omega)\right)<C<\infty
$$

Thus it follows via the standard functional analysis theory, see [16], that

$$
r \in C\left([0, T) ; L_{\omega}^{2}(\Omega)\right)
$$

These estimates show that $r$ remains bounded in the appropriate weighted spaces introduced earlier and thus enables us to state the following theorem.

Theorem 5.3. Consider (1.4) in the TYC system. For positive $r_{0} \in L_{\omega}^{2}(\Omega)$, there exists a unique weak solution $r$ to the system with

$$
\begin{gathered}
r \in C\left([0, T) ; L_{\omega}^{2}(\Omega)\right) \cap L^{\infty}\left(0, T ; L_{\omega}^{2}(\Omega)\right) \cap L^{2}\left(0, T ; H_{0, \omega}^{1}(\Omega)\right), \\
\frac{\partial r}{\partial t} \in L^{2}\left(0, T ; H_{\omega}^{-1}(\Omega)\right) .
\end{gathered}
$$

Furthermore, the solutions are continuous with respect to initial data. 
The uniqueness and convergence result by mimicking the method of proof for Theorem 1.1.

\section{Existence of Global Attractor in Weighted Sobolev Space}

We recall the following spaces from [14], as the natural phase space for our problem:

$$
\begin{gathered}
H=L^{2}(\Omega) \times L^{2}(\Omega) \times L^{2}(\Omega) \times L^{2}(\Omega) \\
Y=H_{0}^{1}(\Omega) \times H_{0}^{1}(\Omega) \times H_{0}^{1}(\Omega) \times H_{0}^{1}(\Omega) \\
X=H^{2}(\Omega) \times H^{2}(\Omega) \times H^{2}(\Omega) \times H^{2}(\Omega)
\end{gathered}
$$

We next state the following definition.

Definition 6.1. Consider a semigroup $S(t)$ acting on a phase space $M$, then the global attractor $A \subset M$ for this semigroup is an object that satisfies

(i) $A$ is compact in $M$.

(ii) $\mathcal{A}$ is invariant, that is, $S(t) \mathcal{A}=\mathscr{A}, t \geq 0$.

(iii) If $B$ is bounded in $M$, then

$$
\operatorname{dist}_{M}(S(t) B, \mathcal{A}) \longrightarrow 0, \quad t \longrightarrow \infty
$$

We showed in [14] that there exists a $(H, X)$ global attractor for the TYC system. That is an attractor that is compact $X$, and attracts bounded subsets in $H$ in the $X$ topology. Furthermore we showed this attractor had finite fractal and Hausdorff dimension. Our goal now is to improve these estimates, on a somewhat different attractor, via the technique of weighted Sobolev spaces. To this end we define

$$
\begin{gathered}
\widetilde{H}=L^{2}(\Omega) \times L^{2}(\Omega) \times L^{2}(\Omega) \times L_{\omega}^{2}(\Omega), \\
\widetilde{Y}=H_{0}^{1}(\Omega) \times H_{0}^{1}(\Omega) \times H_{0}^{1}(\Omega) \times H_{0, \omega}^{1}(\Omega) .
\end{gathered}
$$

Here $\omega$ is the weight as introduced earlier. We will first demonstrate the existence of a $(\widetilde{H}, \widetilde{H})$ attractor for the TYC system. We will then provide estimates for its Hausdorff and fractal dimensions. The following proposition is stated next.

Proposition 6.2. Consider the TYC system, (1.1)-(1.4). There exists a $(\widetilde{H}, \widetilde{H})$ global attractor $\tilde{A}$ for the this system which is compact and invariant in $\widetilde{H}$ and attracts bounded subsets of $\widetilde{H}$ in the $\widetilde{H}$ metric.

The proof follows readily by applying the techniques of [14] to the weighted spaces in question. Recall that there are two essential ingredients to show the existence of a global attractor. The existence of a bounded absorbing set and the asymptotic compactness of the semigroup, see [18]. Thus we will just focus on $r$, as the proof for the other variables is the 
same as in [14]. We will prove the above proposition via two lemmas. The first of these is stated next.

Lemma 6.3. Consider the equation for $r,(1.4)$, in the TYC system. For $r_{0} \in L_{\omega}^{2}(\Omega)$ there exists a bounded absorbing set for $r$ in $L_{\omega}^{2}(\Omega)$.

Proof. Recall, via (5.7), we have

$$
|r(t)|_{2, \omega}^{2} \leq e^{-(C D+\delta) t}\left|r_{0}\right|_{2, \omega}^{2}+\frac{\mu^{2} K^{2} / 2+\mu K}{C D+\delta}, \quad \forall t \geq 0,
$$

Now consider a time $t_{1}$ such that

$$
t_{1}=\max \left(0, \frac{\ln \left(\left|r_{0}\right|_{2, \omega}^{2}\right)}{C D+\delta}\right)
$$

It follows that for any time $t>t_{1}$ the following uniform estimate holds

$$
|r(t)|_{2, \omega}^{2} \leq 1+\frac{\mu^{2} K^{2} / 2+\mu K}{C D+\delta} \leq C
$$

This gives us a bounded absorbing set for $r$ in $L_{\omega}^{2}(\Omega)$.

We next state the following lemma.

Lemma 6.4. The semigroup $S(t)$ for the TYC system, (1.1)-(1.4), is asymptotically compact in $\widetilde{H}$.

Proof. We again demonstrate the proof for $r$. Multiply (5.3) by $-\Delta r e^{\mu x}$ and integrate by parts over $\Omega$ to yield

$$
\begin{aligned}
\frac{1}{2} \frac{d}{d t} \int_{\Omega}|\nabla r|^{2} e^{\mu x} d \mathbf{x} \leq & -D \int_{\Omega}|\Delta r|^{2} e^{\mu x} d \mathbf{x}-\delta \int_{\Omega} \nabla r \cdot \nabla\left(r e^{\mu x}\right) d \mathbf{x} \\
& +\mu \int_{\Omega}\left|\nabla r \frac{\partial r}{\partial t} e^{\mu x}\right| d \mathbf{x} .
\end{aligned}
$$

Now Poincaire's Inequality along with Cauchy-Schwartz imply that

$$
\frac{1}{2} \frac{d}{d t} \int_{\Omega}|\nabla r|^{2} e^{\mu x} d \mathbf{x}+C(D+\delta) \int_{\Omega}|\nabla r|^{2} e^{\mu x} d \mathbf{x} \leq C\left(|\nabla r|_{2}^{2}+\left|\frac{\partial r}{\partial t}\right|_{2}^{2}\right)
$$

However directly from (1.4) and the compact Sobolev embedding of

$$
H^{2}(\Omega) \hookrightarrow H_{0}^{1}(\Omega)
$$


we have

$$
\frac{1}{2} \frac{d}{d t} \int_{\Omega}|\nabla r|^{2} e^{\mu x} d \mathbf{x}+C(D+\delta) \int_{\Omega}|\nabla r|^{2} e^{\mu x} d \mathbf{x} \leq C\left(|\Delta r|_{2}^{2}\right) \leq C
$$

Also, integrating (5.6) in the time interval $\left[t_{1}, t_{1}+1\right]$, we obtain

$$
\int_{t_{1}}^{t_{1}+1}|\nabla r|_{2, \omega}^{2} d t \leq\left|r\left(t_{1}\right)\right|_{2, \omega}^{2}+C
$$

Thus, via a mean value theorem for integrals, we obtain the existence of a time $t_{2} \in$ $\left[t_{1}, t_{1}+1\right]$ such that

$$
\left|\nabla r\left(t_{2}\right)\right|_{2, \omega}^{2} d t \leq C
$$

With this in hand, we can apply Gronwall's Lemma to (6.10), via integration in the time interval $\left[t_{2}, t\right]$ to yield

$$
|\nabla r|_{2, \omega}^{2} \leq e^{-(D+\delta)\left(t-t_{2}\right)}\left|\nabla r\left(t_{2}\right)\right|_{2, \omega}^{2}+C
$$

Thus, there exists a time $t_{3}$ defined by

$$
t_{3}=\max \left(0, t_{2}+\ln \left(\frac{\left|\nabla r\left(t_{2}\right)\right|_{2}^{2}}{C(D+\delta)}\right)\right),
$$

such that for $t>t_{3}$ the following estimate holds uniformly

$$
|\nabla r|_{2, \omega}^{2} \leq 1+C \leq \mathrm{C}
$$

Now, consider any sequence $\left\{r_{0, n}\right\}$, and a sequence of times $\left\{t_{n}\right\}$ such that $t_{n} \rightarrow \infty$. For $n$ large enough we will eventually have $t_{n}>t_{3}$, thus this will yield that for such $t_{n}$, we have

$$
\left|S\left(t_{n}\right) r_{0, n}\right|_{H_{0, \omega}^{1}(\Omega)} \leq C
$$

This follows trivially from (6.15). The standard functional analysis theory, see [17], now implies the existence of a subsequence such that

$$
S\left(t_{n_{j}}\right) r_{0, n} \rightarrow r \quad \text { in } H_{0, \omega}^{1}(\Omega)
$$

However, via the compact Sobolev embedding of

$$
H_{0, \omega}^{1}(\Omega) \hookrightarrow L_{\omega}^{2}(\Omega)
$$


this implies that

$$
S\left(t_{n_{j}}\right) r_{0, n} \longrightarrow r \text { in } L_{\omega}^{2}(\Omega)
$$

This proves the asymptotic compactness in $L_{\omega}^{2}(\Omega)$, and concludes the proof.

Lemmas 6.3 and 6.4 in conjunction prove Proposition 6.2.

Remark 6.5. Note that (1.4), for $r$, is just a diffusion equation with source term. The solutions to (1.4) are functions in $C^{\infty}(\Omega)$. Thus $r$ is trivially in $H^{2}(\Omega)$ and so via the compact Sobolev embedding of $H^{2}(\Omega) \hookrightarrow H_{0}^{1}(\Omega)$, and the form of (1.4), we have the following estimate (which was used earlier)

$$
|\nabla r|_{2}^{2}+\left|\frac{\partial r}{\partial t}\right|_{2}^{2} \leq C|\Delta r|_{2}^{2} \leq C
$$

\section{Improved Estimates for the Global Attractor}

In [14], we derive estimates on the upper bound for the Hausdorff and Fractal dimensions of the global attractor for the TYC system. The estimates are quite crude and are roughly of the order of $K^{3}$, where $K$ is the carrying capacity of the system. Even choosing a modest $K=100$ for our numerical simulations, yields a upper bound of the order of $10^{6}$. This bound is quite impractical. It is of interest for improved numerical and modeling applications to derive much sharper upper bounds, if possible. In this section we derive improved estimates on these dimensions, but for the attractor in $\widetilde{H}$, just described via Proposition 6.2. This is done via projection onto weighted Sobolev spaces. The interested reader is referred to [19] where similar techniques have been used, albeit in the framework for combustion type Stefan problems. We follow the analysis in [19] closely and present details for completeness. For a thorough treatment see [19]. We consider a volume element in the phase space, and try and derive conditions that will cause it to decay. If $\tilde{A}$ is the global attractor of the semigroup $\{S(t)\}_{t \geq 0}$ in $\widetilde{H}$ associated with the Trojan $Y$ Chromosome model, then the trace of the linear operator

$$
\Delta+\delta+F^{\prime}\left(S(\tau) u_{0}\right)
$$

where $F$ is the nonlinear map in (1.1)-(1.4), can be projected onto an $n$ dimensional subspace formally. Let

$$
q_{n}=\limsup _{t \rightarrow \infty} q_{n}(t)
$$

where

$$
\sup _{u_{0} \in \tilde{\mathbb{A}}} \sup _{g_{i} \in H,\left\|g_{g_{i}}\right\|=1,1 \leq i \leq n} \frac{1}{t} \int_{0}^{t} \operatorname{Tr}\left(\Delta U(\tau)-\delta U(\tau)+F^{\prime}\left(S(\tau) u_{0}\right) \circ Q_{n}(\tau)\right) d \tau .
$$


Here, $Q_{n}$ is the orthogonal projection of the phase space $H$ onto the subspace spanned by $U_{1}(t), U_{2}(t), \ldots, U_{n}(t)$, with

$$
U_{i}(t)=L\left(S(t) u_{0}\right) g_{i}, \quad i=1,2, \ldots, n
$$

$L\left(S(t) u_{0}\right)$ is the Frechet derivative of the map $S(t)$ at $u_{0}$. However for our purposes we will choose $Q_{n}$ to be an $n$ dimensional subspace of $H_{0, \omega}^{1}(\Omega) . L\left(S(t) u_{0}\right)$ is the Frechet derivative of the map $S(t)$ at $u_{0}$. We recall the following Lemma from [18].

Lemma 7.1. If there is an integer $n$ such that $q_{n}<0$, then the Hausdorff dimension $d_{H}(\mathcal{A})$ and the fractal dimension $d_{F}(\mathcal{A})$ of $A$ satisfy

$$
d_{H}(\mathcal{A}) \leq n, \quad d_{F}(\mathcal{A}) \leq 2 n .
$$

For the TYC system, $L\left(S(t) u_{0}\right) U_{0}=U(t)=(F(t), M(t), R(t), S(t))$, where $u=$ $(f, m, r, s)$ is a solution to the TYC system. Note that since we are projecting onto a weighted space, we are required to show the existence of solution in such a space. This was demonstrated via Theorem 5.3. Also in our case we will denote $\phi_{j}=\left(\phi_{j}^{1}, \phi_{j}^{2}, \phi_{j}^{3}, \phi_{j}^{4} e^{\mu x}\right)$ to be an orthonormal basis for the subspace $Q_{n}(\tau) H_{0, \omega}^{1}(\Omega)$. Thus we define

$$
\left|\phi_{j}\right|_{2, \omega}^{2}=\left|\phi_{j}^{1}\right|_{2}^{2}+\left|\phi_{j}^{2}\right|_{2}^{2}+\left|\phi_{j}^{3}\right|_{2}^{2}+\left|\phi_{j}^{4} e^{\mu x / 2}\right|_{2}^{2}
$$

The first variational equation for this system is explicitly worked out in [14]. We now estimate

$$
\begin{aligned}
\operatorname{Tr}( & \left.\Delta U(\tau)-\delta U(\tau)+F^{\prime}\left(S(\tau) u_{0}\right) \circ Q_{n}(\tau)\right) \\
& =\sum_{j=1}^{n}\left(\left\langle\Delta \phi_{j}(\tau), \phi_{j}(\tau)\right\rangle_{2, \omega}+\left\langle F^{\prime}\left(S(\tau) u_{0}\right) \phi_{j}(\tau), \phi_{j}(\tau)\right\rangle_{2, \omega}-\delta\left\langle\phi_{j}(\tau), \phi_{j}(\tau)\right\rangle_{2, \omega}\right) \\
& \leq \sum_{j=1}^{n}-4 D\left|\nabla \phi_{j}(\tau)\right|_{2, \omega}^{2}-4 \delta\left|\phi_{j}(\tau)\right|_{2, \omega}^{2}+J_{1}+J_{2}+J_{3} .
\end{aligned}
$$


$J_{1}, J_{2}$, and $J_{3}$ have been worked out in [14]. We recall the details for $J_{1}$ for completeness. Here,

$$
\begin{aligned}
J_{1}= & \sum_{j=1}^{n} \int_{\Omega}\left(f(\tau) \phi_{j}^{2}(\tau) \phi_{j}^{1}(\tau)+m(\tau)\left|\phi_{j}^{1}\right|^{2}\right)\left(1-\frac{f(\tau)+m(\tau)+r(\tau)+s(\tau)}{K}\right) \\
& -f(\tau) m(\tau)\left(\left|\phi_{j}^{1}\right|^{2}+\phi_{j}^{1} \phi_{j}^{2}+\phi_{j}^{1} \phi_{j}^{3}+\phi_{j}^{1} \phi_{j}^{4} e^{\mu x}\right) d \mathbf{x} \\
\leq & \sum_{j=1}^{n} \int_{\Omega}\left(f(\tau) \phi_{j}^{2}(\tau) \phi_{j}^{1}(\tau)+m(\tau)\left|\phi_{j}^{1}\right|^{2}\right) \\
& -f(\tau) m(\tau)\left(\left|\phi_{j}^{1}\right|^{2}+\phi_{j}^{1} \phi_{j}^{2}+\phi_{j}^{1} \phi_{j}^{3}+\phi_{j}^{1} \phi_{j}^{4} e^{\mu x}\right) d \mathbf{x} \\
\leq & \sum_{j=1}^{n} \int_{\Omega}\left(f(\tau)\left(\left|\phi_{j}^{1}\right|^{2}+\left|\phi_{j}^{2}\right|^{2}\right)+m(\tau)\left|\phi_{j}^{1}\right|^{2}\right) \\
& +f(\tau) m(\tau)\left(\left|\phi_{j}^{1}\right|^{2}+\left|\phi_{j}^{1}\right|^{2}+\left|\phi_{j}^{2}\right|^{2}+\left|\phi_{j}^{1}\right|^{2}+\left|\phi_{j}^{3}\right|^{2}+\left|\phi_{j}^{1}\right|^{2}+e^{\mu x}\left|\phi_{j}^{4}\right|^{2}\right) \\
\leq & 4 K^{2} \sum_{j=1}^{n}\left(\left|\phi_{j}\right|_{2, \omega}^{2}\right) .
\end{aligned}
$$

$J_{2}$ and $J_{3}$ are estimated similarly. Thus, we obtain,

$$
\begin{aligned}
\operatorname{Tr}( & \left.\Delta U(\tau)-\delta U(\tau)+F^{\prime}\left(S(\tau) u_{0}\right) \circ Q_{n}(\tau)\right) \\
& \leq-\sum_{j=1}^{n} 4 D\left|\nabla \phi_{j}(\tau)\right|_{2, \omega}^{2}+\mu \int_{\Omega} \nabla \phi_{j}^{4} \phi_{j}^{4} e^{\mu x} d \mathbf{x}-4 \delta\left|\phi_{j}(\tau)\right|_{2, \omega}^{2}+24 K^{2} \sum_{j=1}^{n}\left|\phi_{j}\right|_{2, \omega}^{2} \\
& =-4 D \sum_{j=1}^{n}\left|\nabla \phi_{j}(\tau)\right|_{2, \omega}^{2}+\left(-4 \delta+24 K^{2}\right) \sum_{j=1}^{n}\left|\phi_{j}(\tau)\right|_{2, \omega}^{2}-\frac{\mu^{2}}{2}\left|\nabla \phi_{j}^{4}(\tau)\right|_{2, \omega}^{2} \\
& =-4 D \sum_{j=1}^{n}\left|\nabla \phi_{j}(\tau)\right|_{2, \omega}^{2}+\left(-4 \delta+24 K^{2}-C \mu^{2}\right) \sum_{j=1}^{n}\left|\phi_{j}(\tau)\right|_{2, \omega}^{2} \\
& \leq-4 D \sum_{j=1}^{n}\left|\nabla \phi_{j}(\tau)\right|_{2, \omega}^{2}+\left(24 K^{2}-4 \delta-C \mu^{2}\right) n .
\end{aligned}
$$

This follows via integration by parts on the second term, and property of the eigenfunction $\phi_{j}^{4}$. Now via the generalized Sobolev-Lieb-Thirring inequalities [18] and Lieb-Thirring inequalities for weighted Sobolev spaces [20], we obtain

$$
\sum_{j=1}^{n}\left|\nabla \phi_{j}(\tau)\right|_{2}^{2} \geq K_{1} \frac{n^{5 / 3}}{|\Omega|^{2 / 3}} \int_{\Omega} \omega(\mathbf{x}) d \mathbf{x} .
$$


Here, $K_{1}$ depends only on the shape and dimension of $\Omega$. Thus, we obtain

$$
\begin{aligned}
& \operatorname{Tr}\left(\Delta U(\tau)-\delta U(\tau)+F^{\prime}\left(S(\tau) u_{0}\right) \circ Q_{n}(\tau)\right) \\
& \quad \leq-4 D K_{1} \frac{n^{5 / 3} \int_{\Omega} \omega(x) d \mathbf{x}}{|\Omega|^{2 / 3}}+\left(24 K^{2}-4 \delta-c \mu^{2}\right) n, \quad \tau>0, u_{0} \in \tilde{A} .
\end{aligned}
$$

We now obtain

$$
\begin{aligned}
q_{n}(t) & =\sup _{u_{0} \in \tilde{A}} \sup _{g_{i} \in H,\left\|g_{i}\right\|=1,1 \leq i \leq n} \frac{1}{t} \int_{0}^{t} \operatorname{Tr}\left(\Delta U(\tau)-\delta U(\tau)+F^{\prime}\left(S(\tau) u_{0}\right) \circ Q_{n}(\tau)\right) d \tau \\
& \leq-4 D K_{1} \frac{n^{5 / 3} \int_{\Omega} \omega(x) d \mathbf{x}}{|\Omega|^{2 / 3}}+\left(24 K^{2}-4 \delta-C \mu^{2}\right) n, \quad \forall t>0 .
\end{aligned}
$$

This yields

$$
q_{n}=\limsup _{t \rightarrow \infty} q_{n}(t) \leq-4 D K_{1} \frac{n^{5 / 3} \int_{\Omega} \omega(x) d \mathbf{x}}{|\Omega|^{2 / 3}}+\left(24 K^{2}-3 \delta+\mu\right) \quad n<0,
$$

if the integer $n$ satisfies

$$
n-1<\left(\frac{24 K^{2}-4 \delta-C \mu^{2}}{4 D K_{1} \int_{\Omega} \omega(x) d x}\right)^{3 / 2}|\Omega|<n
$$

Notice that by a specific choice of $\mu$ now we can sharpen the estimates on the trace operator. In particular if we want an upper bound on the Hausdorff dimension, to be of the order $M$, we can choose $\mu$ such that

$$
M^{2 / 3} 4 D K_{1} \int_{\Omega} \omega(x) d x=24 K^{2}-4 \delta-C \mu^{2}
$$

so that we will obtain

$$
n-1<\left(\frac{24 K^{2}-4 \delta-C \mu^{2}}{4 D K_{1} \int_{\Omega} \omega(x) d x}\right)^{3 / 2}, \quad|\Omega|=\left(M^{2 / 3}\right)^{3 / 2}<n .
$$

Via the above analysis, we can now use Lemma 7.1 to obtain the following result. 
Theorem 7.2. Consider the Trojan Y Chromosome model, (1.1)-(1.4). The global attractor $\tilde{A}$ of the system as defined in Proposition 6.2 is of finite dimension. Furthermore given parameters $K, M, \delta$, $D$ and $K_{1}$, if we choose the weight function, and in particular $\mu$, such that the following equation is satisfied

$$
\int_{\Omega} e^{\mu x} d \mathbf{x}+C \mu^{2}=\frac{6 K^{2}-\delta}{M^{2 / 3} D K_{1}}
$$

then, we have the following explicit upper bounds for the Hausdorff and fractal dimensions of the global attractor $\tilde{A}$ :

$$
\begin{aligned}
& d_{H}(A) \leq M+1 \\
& d_{F}(A) \leq 2 M+2
\end{aligned}
$$

\section{Conclusion}

We have demonstrated thus far that the Trojan Y Chromosome model is well posed. We have also shown there exists a global attractor for the system. This validate the TYC strategy as an effective means of eradication of an invasive species. A necessary condition for the existence of a global attractor is the presence of a bounded absorbing set in the phase space. The existence of this implies that indeed the population of invasive species under consideration will be confined to bounded regions after long time, and thus is unable to grow without bound. The analysis of global attractors can be helpful to estimate times to extinction in complex spatial domains. The analysis conducted here determines that for Dirchlet boundary conditions on a connected domain there exists an extinction state as a result of the introduction of feminised supermales $r$.

Furthermore, we have derived upper bounds on the Hausdorff and fractal dimension for the global attractor in the space $\widetilde{H}$. The technique of weighted Sobolev spaces enables us to provide bounds that are of the order of modest order, as opposed to the order of $10^{6}$ that were derived earlier. It should be noted that the improved bounds have been derived via the theoretical construct of weighted sobolev spaces, and it is still a challenge to improve these estimates in the regular Sobolev spaces that were considered in [14], that is without the help of the "weight". However this result has intuitive meaning. Theorem 7.2 tells us there is an inverse relationship between the upper bound $M$ on the Hausdorff dimension for the attractor and $\mu$. Thus there seems to be a scaling of the form $\mu=C / M$. Thus if we want to improve this estimate we will have to increase $\mu$, which physically corresponds to infusing a greater quantity of feminised supermales into the target population of interest, which then would via the mating scenario described earlier, lead to a faster extinction of the female population, and hence ultimately the male population of the invasive species. Thus exploring this scaling further becomes important from a practical point of view. It is also of interest to explore the relation between the attractors $\tilde{A}$ in $\widetilde{H}$, and $\mathcal{A}$ in $H$, particularly for small $\mu$. Various upper semicontinuity methods for attractors, $[17,18]$ could be looked into. These questions are very much on our present agenda.

These estimates are also of numerical importance as most numerical scheme will depend on the number of degrees of freedom in the system. Also note some of the parameters in the system are actually under our control as modellers. Such as the parameter $\mu$. Thus 
understanding fully the dynamics of the system in terms of these parameters is essential if this strategy ever has to be implemented practically. Further questions of well posedness on arbitrary domains and more involved boundary conditions can also be explored. There has been a large interest lately in considering river networks as fractals or dendritic domains. These and related questions are under investigation. Further more we also believe that we can consider questions regarding existence of mild and strong solutions to the system, which can be demonstrated if further regularity of the solutions can be shown. This question is also currently under investigation. The other more practical question is to consider other forms of the parameter $\mu$. That is to consider what might happen if feminised supermales were added not at a constant rate but perhaps periodically. This leads to considering the question of a time dependent $\mu$. It might also be feasible to look at a stochastic $\mu$ term, and thus bring in tools from stochastic PDE's to further inform our analysis.

In short, we would like to conclude by reiterating that invasive species pose a clear and present danger to both the ecosystems they reside in and the regional and global economies they affect. They spread quickly and are difficult to eradicate. We believe or research efforts to this end, despite in a nascent stage, will be of pragmatic and financial value to both ecologists, government and industries in the long run.

\section{References}

[1] J. Hill and C. Cichra, "Eradication of a reproducing population of Convict Cichlids, Cichlasoma nigrofasciatum (Cichlidae) in North-Central Florida," Florida Scientist, vol. 68, no. 2, pp. 65-74, 2005.

[2] P. Shafland and K. Foote, "A reproducing population of Serrasalmus humeralis Valenciennes in southern Florida," Florida Scientist, vol. 42, no. 4, pp. 206-214, 1979.

[3] D. Pimentel, R. Zuniga, and D. Morrison, "Update on the environmental and economic costs associated with alien-invasive species in the United States," Ecological Economics, vol. 52, no. 3, pp. 273-288, 2005.

[4] J. B. Gutierrez, Mathematical analysis of the use of trojan sex chromosomes as means of eradication of invasive species, Ph.D. thesis, Florida State University, Tallahassee, Fla, USA, 2009.

[5] V. P. Palace, R. E. Evans, K. Wautier et al., "Induction of vitellogenin and histological effects in wild fathead minnows from a lake experimentally treated with the synthetic estrogen, ethynylestradiol," Water Quality Research Journal of Canada, vol. 37, no. 3, pp. 637-650, 2002.

[6] J. B. Gutierrez and J. L. Teem, "A model describing the effect of sex-reversed YY fish in an established wild population: the use of a Trojan Y chromosome to cause extinction of an introduced exotic species," Journal of Theoretical Biology, vol. 241, no. 2, pp. 333-341, 2006.

[7] A. B. J. Bongers, B. Zandieh-Doulabi, C. J. J. Richter, and J. Komen, "Viable androgenetic YY genotypes of common carp (Cyprinus carpio L.)," Journal of Heredity, vol. 90, no. 1, pp. 195-198, 1999.

[8] A. Felip, A. Fujiwara, W. P. Young et al., "Polymorphism and differentiation of rainbow trout Y chromosomes," Genome, vol. 47, no. 6, pp. 1105-1113, 2004.

[9] K. B. Davis, B. A. Simco, C. A. Goudie, N. C. Parker, W. Cauldwell, and R. Snellgrove, "Hormonal sex manipulation and evidence for female homogamety in channel catfish," General and Comparative Endocrinology, vol. 78, no. 2, pp. 218-223, 1990.

[10] L. A. P. Carrasco, D. J. Penman, and N. Bromage, "Evidence for the presence of sex chromosomes in the Nile tilapia (Oreochromis niloticus) from synaptonemal complex analysis of $X X, X Y$ and $Y Y$ genotypes," Aquaculture, vol. 173, no. 1-4, pp. 207-218, 1999.

[11] J. J. Nagler, J. Bouma, G. H. Thorgaard, and D. D. Dauble, "High incidence of a male-specific genetic marker in phenotypic female chinook salmon from the Columbia River," Environmental Health Perspectives, vol. 109, no. 1, pp. 67-69, 2001.

[12] M. A. Hurley, P. Matthiessen, and A. D. Pickering, "A model for environmental sex reversal in fish," Journal of Theoretical Biology, vol. 227, no. 2, pp. 159-165, 2004.

[13] J. B. Gutierrez, M. K. Hurdal, R. D. Parshad, and J. L. Teem, "Analysis of the trojan y chromosome model for eradication of invasive species in a dendritic riverine system," Journal of Mathematical Biology. Revision Submitted. 
[14] R. D. Parshad and J. B. Gutierrez, "On the Global Attractor of the Trojan Y Chromosome Model," Communications on Pure and Applied Analysis, vol. 10, no. 1, pp. 339-359, 2011.

[15] B. Wang and S. Lin, "Existence of global attractors for the three-dimensional Brinkman-Forchheimer equation," Mathematical Methods in the Applied Sciences, vol. 31, no. 12, pp. 1479-1495, 2008.

[16] R. Temam, Navier-Stokes Equations and Nonlinear Functional Analysis, Society for Industrial and Applied Mathematics, Philadelphia, Pa, USA, 1985.

[17] J. C. Robinson, Infinite-Dimensional Dynamical Systems: An Introduction to Dissipative Parabolic PDEs and the Theory of Global Attractors, Cambridge Texts in Applied Mathematics, Cambridge University Press, Cambridge, UK, 2001.

[18] R. Temam, Infinite-Dimensional Dynamical Systems in Mechanics and Physics, Applied Mathematical Sciences, Springer, New York, NY, USA, 1998.

[19] M. Frankel and V. Roytburd, "Dynamics of thermally-insulated nonequilibrium Stefan problem," Journal of Evolution Equations, vol. 7, no. 2, pp. 317-345, 2007.

[20] K. Tachizawa, "Weighted Sobolev-Lieb-Thirring inequalities," Revista Matemática Iberoamericana, vol. 21, no. 1, pp. 67-85, 2005. 